\title{
Calendar of Short Courses \& Workshops
}

\author{
Gang Wang \\ Research and Development Center for Functional Crystals, Institute of Physics, Chinese Academy of Sciences, \\ No. 8 Nansanjie, Zhongguancun, Haidian District Beijing 100190, China \\ gangwang@iphy.ac.cn
}

\section{4-6 January 2016}

First BioStruct-X Mediterranean Macromolecular Crystallography Workshop Technion, Israel [Info: http:// tcsb-biox-wksp.net.technion.ac.il/]

\section{2-25 February 2016}

International School and Conference on Biological Materials Science Potsdam, Germany [Info: http://bioins pired.inventum.de/home/]

\section{February-4 March 2016}

Thirty-Sixth Berlin School on Neutron Scattering Berlin, Germany [Info: http://www.helmholtz-berlin.de/events/neu tronschool/]

\section{9-11 March 2016}

Ninth International Workshop on X-Ray Radiation Damage to Biological Crystalline Samples Lund, Sweden [Info: http://indico.maxlab.lu.se/event/67/]

\section{5-29 April 2016}

Practical X-Ray Fluorescence Newtown Square, Pennsylvania, USA [Info: http://www.icdd.com/education/ xrf.htm]

\section{6-20 May 2016}

Fundamentals of X-Ray Powder Diffraction Newtown Square, Pennsylvania, USA [Info: http://www.icdd.com/edu cation/xrd.htm]

\section{3-27 May 2016}

Advanced Methods in X-Ray Powder Diffraction Newtown Square, Pennsylvania, USA [Info: http://www.icdd.com/edu cation/xrd.htm]

27 May-5 June 2016

High-Pressure Crystallography: Status Artis and Emerging Opportunities Erice, Italy [Info: http://crystaler ice.org/2016/]
7-10 June 2016

International Workshop on Phase Retrieval and Coherent Scattering Saint Malo, France [Info: http://www.synchrotronsoleil.fr/portal/page/portal/Soleil/ToutesActualites/Workshops/ 2016/COHERENCE-2016/Accueil]

27 June-2 July 2016

International School on Fundamental Crystallography with Applications to Electron Crystallography University of Antwerp, Antwerp, Belgium [Info: http://www.crystallog raphy.fr/mathcryst/antwerp2016.php]

\section{4-8 July 2016}

Third International School on Aperiodic Crystals Antwerp, Belgium [Info: https://www.uantwerpen.be/en/ summer-schools/aperiodic-crystals/]

\section{1-7 August 2016}

Sixteenth International Summer School on Crystal Growth (ISSCG-16) Lake Biwa, Shiga, Japan [Info: http:// www.iccge18.jp/isscg16/]

\section{2-26 August 2016}

Powder Diffraction School: Modern Synchrotron Methods Villigen, Switzerland [Info: https://indico.psi.ch/conference Display.py?confId=2592]

\section{3-26 August 2016}

International School on Charge and Spin Densities: From Experimental Determination to Interpretation Nancy, France [Info: http://crm2.univ-lorraine.fr/lab/education/congres/ ecm30-congress-satellite-school/]

25 March-2 April 2017

Sixteenth BCA/CCG Intensive Teaching School in X-Ray Structure Analysis Trevelyan College, Durham, UK [Info: http://community.dur.ac.uk/durham.x-ray-school/staff. htm] 